\title{
Smart design of fiber optic surfaces for improved plasmonic biosensing
}

\section{Iulia Arghir, Filip Delport, Dragana Spasic and Jeroen Lammertyn}

KU Leuven, Department of Biosystems, MeBioS-Biosensor Group, Willem de Croylaan 42, 3000 Leuven, Belgium

\section{Abstract}

Although the phenomenon of surface plasmon resonance (SPR) is known for more than a century now, traditional prism-based SPR platforms have hardly escaped the research laboratories despite being recognized for the sensitive and specific performance. Significant efforts have been made over the last years to overcome their existing limitations by coupling the SPR phenomenon to the fiber optic (FO) technology. While this platform has been promoted as cost-effective and simpler alternative capable of handling label-free bioassays, quantification and real-time monitoring of biomolecular interactions, examples of its applicability in sensing and biosensing remain to date very limited. The FO-SPR system is still in development and requires further advancements for reaching the stability and sensitivity of the benchmark SPR systems. Among existing strategies for device improvement, those based on modifying the FO tips using nanomaterials are mostly studied. These small-scale objects provide a wide range of possibilities for alternating the architecture of the FO sensitive zone, enabling also unique effects such as localized SPR (LSPR). This mini-review summarizes the latest innovations in the fabrication procedures which use nanoparticles or other nanomaterials, aiming at FO-SPR technology performance improvements, as well as addition of new device features and functionalities.

\section{Contents}

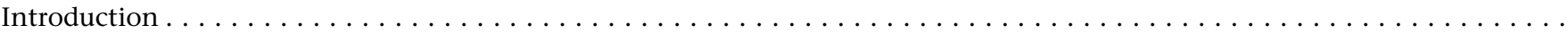

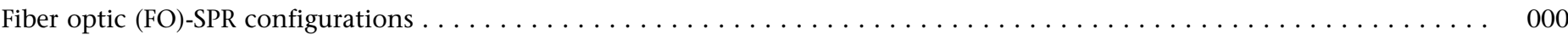

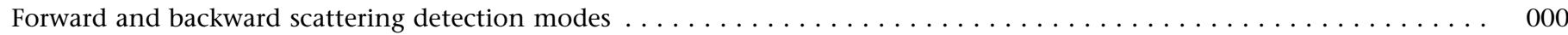

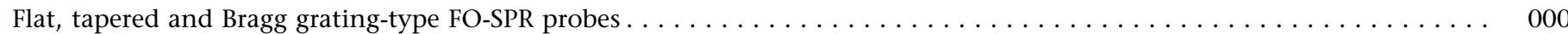

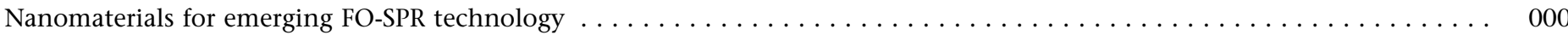

Nanoparticles as amplification labels in the FO-SPR bioassays $\ldots \ldots \ldots \ldots \ldots$

Texturing the FO-SPR surfaces with metallic nanoparticles $\ldots \ldots \ldots \ldots \ldots \ldots$

FO-SPR probes with lithographically patterned nanostructures. $\ldots \ldots \ldots \ldots \ldots$

Nanoparticles coupled into microstructured FO-SPR probes $\ldots \ldots \ldots \ldots \ldots$

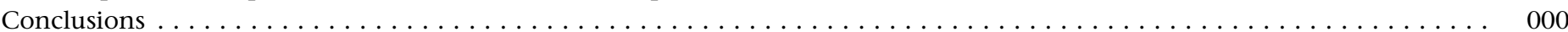

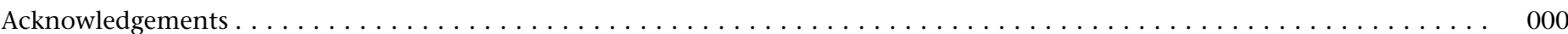

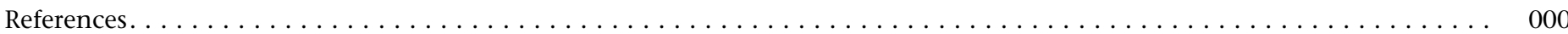




\section{Introduction}

The first proof of surface plasmon resonance (SPR) dates back in 1902 when Wood observed a pattern of dark and bright bands in the reflected light coming from a polarized light wave incident to a diffraction grating built on top of a mirror surface [1]. Despite various attempts of addressing this optical occurrence $[2,3]$, the first complete explanation of the effect was done more than 60 years later by Otto [4] and Kretschmann [5], when they reported the excitation of surface plasmons (SPs). Since then, the physical phenomenon of SPR has found its way into many research areas of biology, biochemistry and medicine, because it provides a labelfree alternative for the detection and quantification of biomolecular interactions, allowing direct access to the kinetic parameters and binding constants. Today, the SPR technology is a well-established optical sensing technique with many proven applications in medical diagnostics [6] and life-science research [7,8], where the SPR sensors are mostly used to measure real-time interactions between proteins, lipids, nucleic acids or even low molecular weight molecules such as drugs [9].

SPs are coherent electron oscillations forming along the interface between a thin metallic film (typically gold-Au or silver-Ag) and a dielectric medium, which usually represents the analyzing environment (typically a liquid) [10]. The SPs are excited when guiding a polarized light toward this interface. When the energy and momentum of both, the incident light and SPs match, a resonance condition results in the omission of part of the reflected light intensity, also referred to as a sharp spectral dip. This resonance condition is strongly dependent on the refractive index (RI) of the dielectric medium. Therefore, even very small changes in the RI value (potentially associated with: metal surface modifications, slight variations in the composition and concentration of the analyzing sample, or various biomolecular binding events occurring at the metal/dielectric interface) will produce a measurable shift in the resonance dip.

In order to excite the SPs, the incident light reaching the metal/ dielectric interface has to be modulated using a light propagation medium such as an optical prism, which generates total internal reflection (TIR) [11]. The first prism-based system used for SPs excitation was the Otto configuration with the dielectric medium confined between the prism base and a thin Au layer [4]. This configuration was rarely used in SPR biosensing due to the construction difficulties related to the small gap $(\sim 1 \mu \mathrm{m})$ between the prism and the metal. Few years later, the Kretschmann system became the most famous SPR configuration, with the Au film (between 10 and $100 \mathrm{~nm}$ thick) being directly deposited on the prism base and further exposed to the dielectric medium [5]. Although these classical SPR platforms were substantially improved during the last decade [12-14] (i.e. BiaCore system [15]), they still present several shortcomings such as large sizes and the necessity to use expensive optical equipment and precise mechanical components. In addition, the target solution has to be typically handled using advanced pump-driven microfluidic schemes, which make these platforms difficult to use outside the research centers and unsuitable for investigation of liquid samples in small quantities.

Many of the presented drawbacks associated with the traditional SPRs have been compensated by replacing the prism with an optical fiber, in a new family of devices called fiber optic (FO)-SPR sensors [16]. Here, the FO serves as a waveguide for the incident light and it is typically coated with a thin Au layer. The plasmonic interface is then enabled when exposing such probes to the desired dielectric environment. One important advantage is that the entire system can be substantially miniaturized making the FOSPR sensors highly portable for measurements outside the laboratory environment [17]. Moreover, the FO-SPRs exhibit other interesting features as well, such as: low-cost components, capacity for multichannel performance required in high-throughput screening applications and the possibility for improved sensitivity through the incorporation of various nanomaterials into bioassays, an approach not feasible with the classical microfluidics-based SPR systems [18].

Despite all these advantages, the FO-SPR setup still requires some improvements in order to reach the stability, sensitivity and limit of detection (LOD) of the traditional prism-based SPRs [19]. Since the mid-nineties when Villuendas and Palayo were among the first proposing a FO-SPR sensor design [20], a wide range of strategies were constantly employed by the researchers, aiming to improve the overall performance of the FO-SPR sensing platform [21]. Among others, it has been shown that by: (i) accurately controlling the chemical interactions at the sensor surface [22], (ii) improving the adhesion of the $\mathrm{Au}$ thin film deposited on the FO substrate [23], (iii) implementing signal amplification protocols using $\mathrm{Au}$ or magnetic nanoparticles (NPs) [24], (iv) structuring the sensor surface using various nanomaterials [25] or (v) well-controlled nanostructuring techniques [26], the stability, specificity and/or sensitivity of the FO-SPR device can be further improved. While the first two approaches are well covered within previous interesting reviews on this topic $[11,27]$, the latter ones involving the use of NPs or other nanomaterials are still in the early stages of development, challenging continuously the scientific community. Besides their use for increasing the overall performance of the FO-SPR sensors, these nano-scale objects enable also unique effects such as localized surface plasmon resonance (LSPR), where the localized SPs (LSPs) are excited on the sub-wavelength-sized metallic nanostructures. In this context, the strong electromagnetic (EM) field confinement of LSPs reduces the penetration depth of the evanescent field into the surrounding dielectric medium. As a direct consequence, the biomolecules attached to the nanostructure surface take a much larger fraction of the evanescent field, determining an exceptional sensitivity for the detection of tiny biomolecules in low amounts [12], but possibly compromising it for larger biomolecules. While the LSPR biosensing with classical planar substrates recently found its way on the market (i.e. Insplorion XNano [28]), its implementation onto the curved FO geometry has not yet escaped the research laboratories with many new construction possibilities left to be further explored.

This mini-review provides a brief overview of the most commonly used FO-SPR architectures with the focus on recent approaches for improving both SPR and LSPR biosensing technology. First, specific aspects related to the physical construction of the FO-SPR probes are discussed in relation to the expected performance and/or achieved functionality of the final device. The second part reviews several strategies for further modifying the FO tips of the traditional FO-SPR probes. Most of the presented approaches are based on decorating the classical FO tips with 
various NPs or other nanomaterials, targeting on one hand addition of new device functionalities, and on the other hand performance improvements and/or LSPR sensing capabilities.

\section{Fiber optic (FO)-SPR configurations}

\section{Forward and backward scattering detection modes}

Whereas the FO-SPR probes can be prepared using different approaches, they are mainly manufactured either (i) by etching the intermediate cladding to expose the FO core or (ii) by surface modification of one of the FO ends [29].

The first approach entails an etching step of the FO cladding in order to expose its core at an intermediate point between the two ends. In this configuration, one FO end is used for propagating the exciting light wave while the reflected light is then collected by the other FO end. This detection scheme is known as "forward scattering" mode (Fig. 1A). The first report on this type of probe dates back to 1988 where, after the cladding etching, a thin Au layer $(\sim 50 \mathrm{~nm})$ was deposited on the FO core exposed area [29]. The modified portion of the FO was immersed in the target solution while the changes in the RI of the reflected light were monitored during the sensing event. Although this fabrication method was extensively used for several years to create various FO-based plasmonic sensors and biosensors, it was reported later as relatively difficult to implement in a reproducible manner, due to the necessity of localized polishing and/or dissolution of the FO cladding, which substantially decreases the FO mechanical elasticity and strength $[30,31]$.

In contrast, the second fabrication method relies on the complete removal of the FO jacket and cladding at one end of the FO, followed by the deposition of a thin $(\sim 50 \mathrm{~nm})$ metallic layer, typically $\mathrm{Au}$ [32] or $\mathrm{Ag}$ [33]. In this case, the modified tip of the resulted FO-SPR probe is immersed in the sample solution while both, the excitation and detection of the reflected light occur at the FO distal end. This detection mode is called "backward scattering" (Fig. 1B). The first FO-SPR design based on a modified FO tip was developed in 1993 [34]. Since then, this configuration has been widely implemented and numerous similar FO-SPR devices have been introduced in an attempt to enhance the performance of the entire sensing platform $[35,36]$. The FO-SPR probes fabricated in this manner are extremely versatile, more suitable for in-situ sensing and more reliable because the exposed FO section is guaranteed to be strain and/or bend free, and thus much less susceptible to stress-induced break.

Moreover, the sensors with "backward scattering" detection mode are particularly promising because their special configuration allows application of an extremely wide range of techniques for modifying the FO tips. This review will therefore mainly focus on these FO-SPR probes, summarizing the most reliable strategies currently used to modify their tips in order to obtain sensors with new functionalities or simply with improved performance.

\section{Flat, tapered and Bragg grating-type FO-SPR probes}

A standard FO-SPR sensing probe with "backward scattering" detection mode can be manufactured by cutting first a small FO piece, with a consistent length of several centimeters. Then, a sensitive zone is constructed by mechanically removing the FO jacket at one side and chemically etching the polymer cladding to expose the FO silica core. The latter is finally coated with the desired thin plasmonic film.

Jorgenson and Yee proposed one of the first fabrication methodologies, by dissolving the cladding around the tip of a multimode FO and depositing a thin Au film $(\sim 55 \mathrm{~nm})$ just on the

\section{A. Forward scattering detection mode}

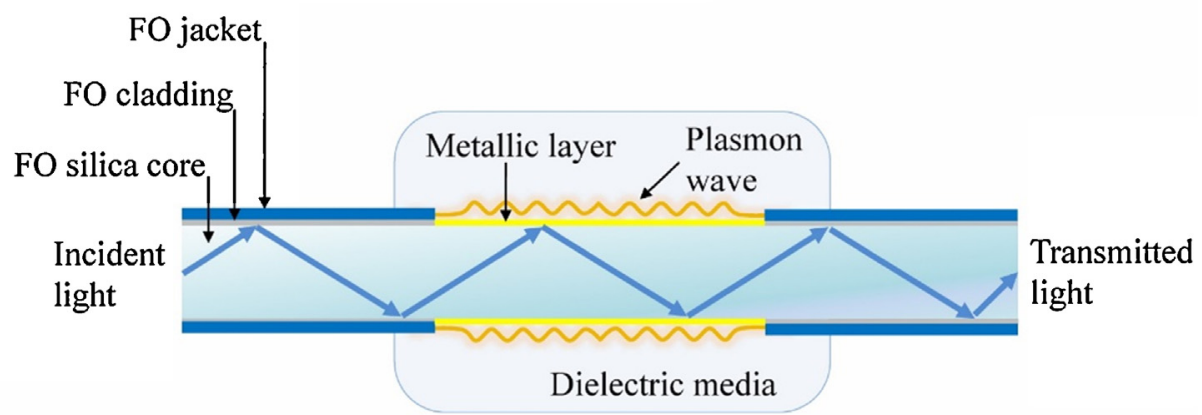

B. Backward scattering detection mode

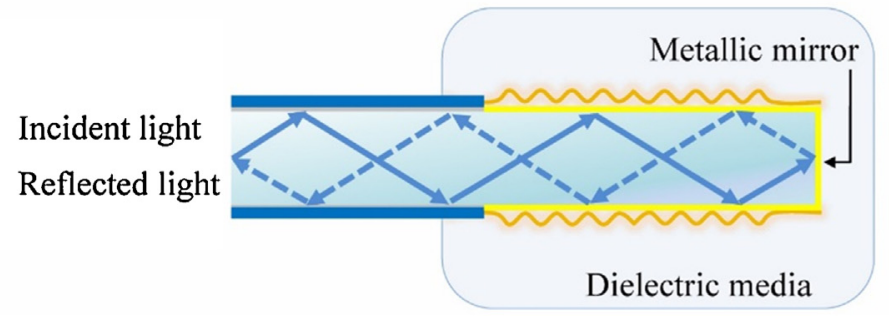

FIG. 1

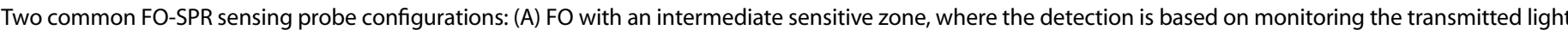

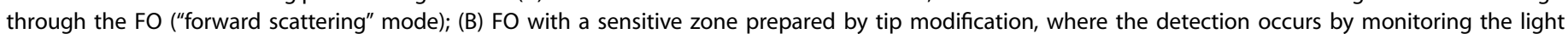
reflected back by the FO "mirror-like" prepared tip ("backward scattering" mode). 
circular side of the etched FO tip by electron-beam evaporation [37]. This step was followed by a second deposition of a $300 \mathrm{~nm} \mathrm{Ag}$ mirror on the flat FO tip. The authors demonstrated that the dynamic range of this sensor could be extended between 1.00 and 1.40 refractive index units (RIU) by the deposition of a high RI material on top of the $\mathrm{Au}$ layer, like zirconium dioxide $\left(\mathrm{ZrO}_{2}\right)$. Furthermore, they reached even 1.70 RIU when replacing the FO standard silica core with a sapphire one. Pollet et al. proposed later a much simpler FO-SPR design, by coating the tip (with a $1 \mathrm{~cm}$ long SPR sensitive zone) of a multi-mode FO (400 $\mu \mathrm{m}$ in diameter) with a thin and consistent $\mathrm{Au}$ layer $(\sim 50 \mathrm{~nm})$ using the sputtering technology [32]. The sensitivity of this sensor was $1722 \mathrm{~nm} / \mathrm{RIU}$ [38]. This simplified design was reported as the first one used for label-free measuring of both DNA hybridization and DNA-protein interactions, with demonstrated LODs in the high nM range. Furthermore, the same authors demonstrated improvements in the LOD of this particular device when implementing labeling approaches as discussed in Section 3.1. Using the same configuration, Suzuki and co-workers studied the dependence of the device performance on the Au layer thickness. The results showed a maximum sensitivity of $1557 \mathrm{~nm} / \mathrm{RIU}$ (in the RI range of 1.3330-1.3469) achieved for an optimized $\mathrm{Au}$ thickness of $\sim 65 \mathrm{~nm}$ [39].

An interesting approach currently explored by the researchers is to replace the classical flat tip FO with a tapered FO (TFO) where the tip shape is modified prior to the metal deposition. By reshaping the FO tip, additional optimizations of the EM field distribution across the plasmonic interface are feasible [40], possibly bringing further improvements in the working wavelength range of these FO-SPR sensors. However, FO tips polished at a defined angle relative to the FO longitudinal axis and then covered with either a thin Ag [41] or $\mathrm{Au}$ [16] film did not give a spectacular increase in performance. Only when selectively tapering the FO distal end at different angles and in several forms (i.e. trapezoid or pyramidal shapes), the resonance wavelength was either redshifted for more than $100 \mathrm{~nm}$ or blue-shifted for more than $30 \mathrm{~nm}$, increasing thus the dynamic range of accessible RI values [42]. The size of the red-shift was positively correlated to the tapering angle, whilst the blue-shift was mainly influenced by the tapering form. The authors demonstrated in this way that an optimized shape and a carefully prepared FO tip could increase the sensitivity by a factor of 4 [36]. Furthermore, a conically tapered FO with a tip of $100 \mathrm{~nm}$ showed a 4000 times higher sensitivity when used with a fixed excitation laser $(650 \mathrm{~nm})$ for exciting the SPs [43]. Following the same idea, a near-infrared excited FO-SPR sensor was designed [44]. Among several tested relative angles of FO tip tapering, the best performating device exhibited a sensitivity of $2.4 \times 10^{4} \mathrm{~nm} / \mathrm{RIU}$, about one order of magnitude higher compared to the $1.9 \times 10^{3} \mathrm{~nm} / \mathrm{RIU}$ obtained for the flat (non-tapered) FO tip.

A promising fabrication strategy was also elaborated by Shevchenko and co-workers $[45,46]$. They inscribed a tilted Bragg grating inside the core of a single-mode FO using the phase-mask technique [47]. After jacket removal, the FO was directly exposed to intense ultraviolet (UV) laser light $(248 \mathrm{~nm})$ through a grooved glass plate, before covering the surface of the FO's cladding with a thin $\mathrm{Au}$ film of $50 \mathrm{~nm}$ (Fig. 2) [48]. In such a configuration, the sensor generated a "comb-like" spectrum of SPR resonances, due to an individual coupling of the core mode light to a multitude of

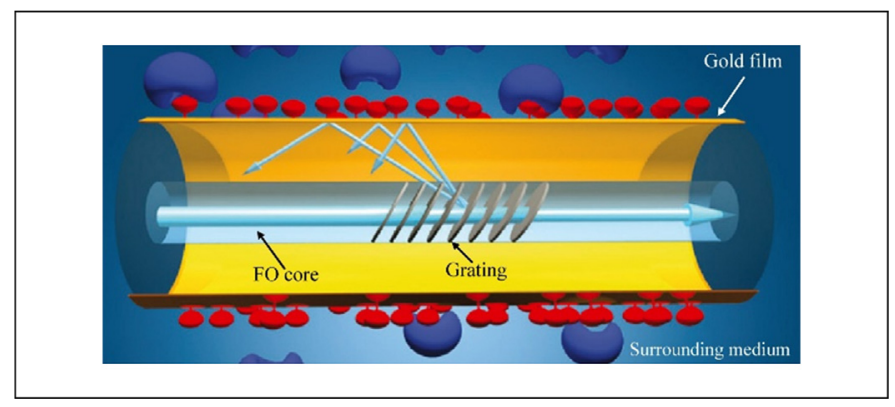

FIG. 2

Schematic design of a tilted Bragg grating-based FO-SPR sensing probe. Adapted with permission from [45]. Copyright (2011) American Chemical Society.

cladding modes, providing as well a mechanism to eliminate the effect of temperature cross-sensitivity from the system's readout. Those resonances that were phase-matched to the SPR might provide sensitivities up to $1000 \mathrm{~nm} / \mathrm{RIU}$. Gratings as long as $15 \mathrm{~mm}$, with linewidths of $0.1 \mathrm{~nm}$, could thus yield impressive figures of merit (FOM-defined as the ratio between the sensitivity and the corresponding full width at half maximum-FWHM), close to 10,000 . The device was used for real-time monitoring of aptamers (synthetic DNA sequences) implementation on the FO surface, as well as for the detection of target proteins (i.e. thrombin) in different solutions including serum [45]. Theoretical simulations were also carried out on a similar device architecture by systematically varying both, the grating length and the Au layer thickness, targeting optimizations of SPR line width, absorption depth and sensitivity [49]. The optimized values were $2 \mathrm{~mm}$ for the grating length and $50 \mathrm{~nm}$ for the thin Au film [49].

Other existing reviews provide already a comprehensive overview of the currently investigated geometries in the FO-SPR probes fabrication [11,27]. One evident overall conclusion is that an optimized FO-SPR configuration leads to an enhanced EM field and spectral resolution, which are both desired, as they promote a better LOD and sensitivity of the sensor. Nevertheless, certain design limitations prevent these traditional configurations to be additionally improved or to complete the way toward LSPR sensing, and other technological approaches are necessary, some of them being presented further in this review.

\section{Nanomaterials for emerging FO-SPR technology} Nanoparticles as amplification labels in the FO-SPR bioassays

Although there are several important advantages of the so far presented FO-SPR sensor configurations, these systems still offer lower sensitivities with relatively modest LODs, when compared with the classical SPR devices. Both parameters can be improved by amplifying the SPR response with smart labeling techniques using $\mathrm{Au}$, silica and/or magnetic NPs [50].

$\mathrm{Au}$ NPs have great potential as transducers and signal amplification labels in biosensors design due to their plasmonic, catalytic, scattering or quenching properties [51]. When used as SPR signal amplification labels, they improve the sensitivity not only due to the increased binding mass, but also due to the increased perturbation of the evanescent field [52], generating strong LSPR effects. $\mathrm{Au}$ NPs of different sizes and shapes, functionalized with various bioreceptors [19], being commonly antibodies [50] or lately also 
aptamers [52,53], could be successfully utilized in "sandwich-like" bioassays subsequently implemented onto the traditional FO-SPR probes (presented in Section 2.2). Furthermore, they have proven to be very efficient labeling agents for SPR-based studies of oligonucleotide interactions, allowing real-time monitoring of DNA hybridization and melting, thus paving the way toward specific applications like fast DNA quantification or genomic screening. In this context, Pollet et al. [54] reported for the first time the FO-SPR signal enhancement using Au NPs, being followed later by Knez et al. $[55,56]$. They proposed an ultra-sensitive genetic screening tool using DNA-functionalized Au NPs bound to the sensor surface in the presence of a single-stranded DNA target. Moreover, using the same system, fast and direct detection of single-point mutations (SNPs) in the target DNA was possible, by monitoring the changes in the melting temperature of NPs-DNA complexes (Fig. 3). In their studies, the authors used a set of bacterial and other DNA targets with different SNPs demonstrating a good accuracy of the Au NPs-enhanced FO-SPR sensor, with observed LODs in the $\mathrm{nM}$ range, three orders of magnitude below the classical high-resolution melting-based screening. Following a similar strategy, the same FO-SPR platform was used also in combination with silica NPs as signal enhancers [57]. The sensor was placed inside a standard polymerase chain reaction (PCR) thermocycler to accurately monitor the hybridization reactions between the DNA immobilized on the sensor surface and the DNA strands of different lengths linked to the silica NPs. A novel FO-SPR sensing platform was proposed by Hsieh et al. using the Au NPs as fluorescent labels [58]. A photomultiplier was used to collect the fluorescence signal for guiding it through the FO with high efficiency. This FO-SPR system could detect mouse immunoglobulin $\mathrm{G}$ (IgG) from buffer solutions with concentrations as low as $1 \mathrm{pg} /$ $\mathrm{mL}$ (7 $\mathrm{fM})$, providing thus a very sensitive platform.

Excellent FO-SPR signal amplification has been obtained as well with magnetic NPs, as they can pre-concentrate the target from the

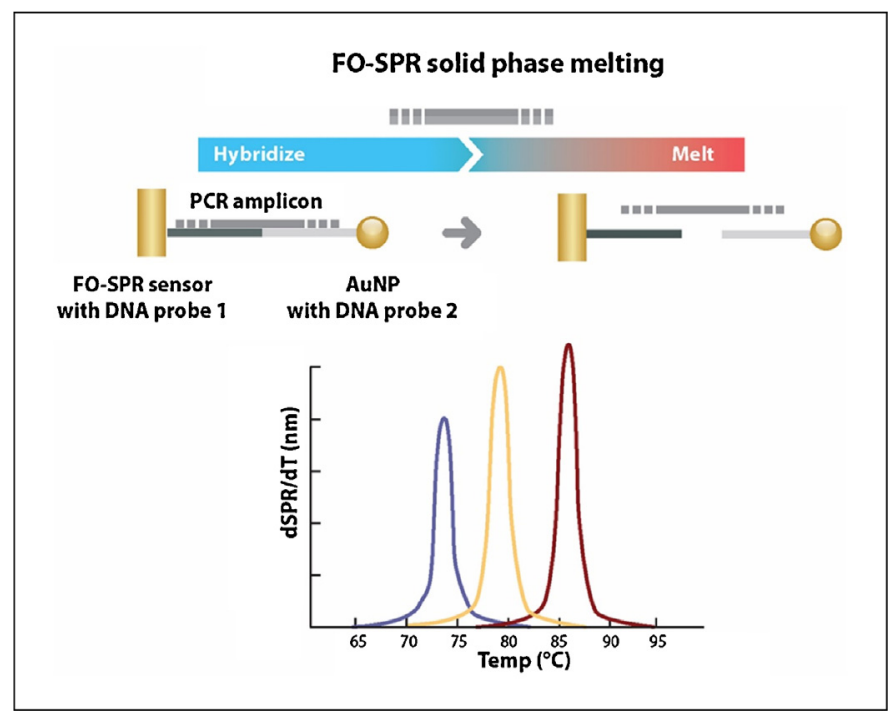

FIG. 3

The concept of genetic screening using FO-SPR technology, where DNAfunctionalized Au NPs are bound first to the sensor surface in the presence of a target and then changes in the melting temperature of DNA-Au NPs complexes are monitored, being associated with the variations in the target DNA sequences. Reprinted with permission from [56]. Copyright (2013) American Chemical Society. sample matrix and thereby further improve the SPR response not only by increasing the sensitivity but also by increasing the specificity of the sensor. Such an approach was first tested, by implementing a "sandwich-like" assay for the detection of Ara h1 peanut allergen in a complex food matrix (i.e. chocolate) [59]. The authors have shown that by using magnetic NPs as a secondary label, the LOD was substantially improved with at least two orders of magnitude (from 9 to $0.09 \mu \mathrm{g} / \mathrm{mL}$, Fig. 4 ). When the results were compared with a commercially available ELISA kit, the NPs-enhanced FO-SPR sensor demonstrated similar performance in terms of LOD, but a superior dynamic range and a much faster analysis time.

Although the labeling techniques using NPs are extremely promising, either for adding new functionalities to the FO-SPR sensor or just for amplifying the sensor output signal, the strategies are not so extensively applied yet in FO-SPR technology. Besides, these approaches tend to be superseded by the much more advanced methods for precisely localizing the NPs and controlling their aspect and size, as discussed further.

\section{Texturing the FO-SPR surfaces with metallic nanoparticles}

One possibility for boosting the FO-SPR performance is to modify the FO tip by directly texturing its surface with various nanomaterials (i.e. NPs, nanorods), an approach well-established for the traditional SPR systems with planar substrates [12]. This strategy can potentially enable LSPR sensing capacity and may be achieved in two ways: (i) by coating the FO tips with the desired thin metal film and then performing special surface treatments for altering its morphology or (ii) by directly coating metallic nanostructures on the FO sensitive area.

The first report about modifying a FO tip by surface pre-treatment was written by Mullen and Carron, although the obtained device was used at that time only for surface-enhanced Raman scattering (SERS) measurements [60]. The configuration consisted of a mechanically roughened $600 \mu \mathrm{m}$ core FO tip, which was first treated with ammonium hydroxide $\left(\mathrm{NH}_{4} \mathrm{OH}\right)$ solution followed by depositing Ag nano-islands on the tip by thermal evaporation. Only later, the potential to use such a device as LSPR sensor was demonstrated. The Ag islands were replaced with a thin Au film, which was subsequently annealed in controlled conditions in order to reshape its morphology and to get randomly distributed $\mathrm{Au}$ NPs on the sensor surface [61]. The authors obtained in this way a spectral resonance dip around $535 \mathrm{~nm}$ in air, which was further shifted when the probe was immersed in other media, generating a sensitivity of $110 \mathrm{~nm} / \mathrm{RIU}$.

In the second approach, the Au NPs were directly deposited on the FO-SPR surface [62]. The method was based on the pre-silanization of the FO tip in order to directly attract chemically synthesized Au NPs. This procedure resulted in $20 \%$ coverage of the FO tip and a resolution of $2 \times 10^{-5}$ RIU. Jeong et al. demonstrated further improvements in the sensor's sensitivity by controlling the Au NPs density on the surface (Fig. 5) [25]. They tested this device for detecting the antibody-antigen reaction of interferon-gamma (IFN- $\gamma$ ), reporting first a LOD of $10 \mathrm{pg} / \mathrm{mL}$, and later even $2 \mathrm{pg} /$ $\mathrm{mL}$ for the same immunoassay, as a consequence of improved synthesizing protocol for the Au NPs and the subsequent immobilization step on the FO surface. Furthermore, they reached $1 \mathrm{pg} /$ $\mathrm{mL}$ when using this FO-LSPR sensor for the detection of the 


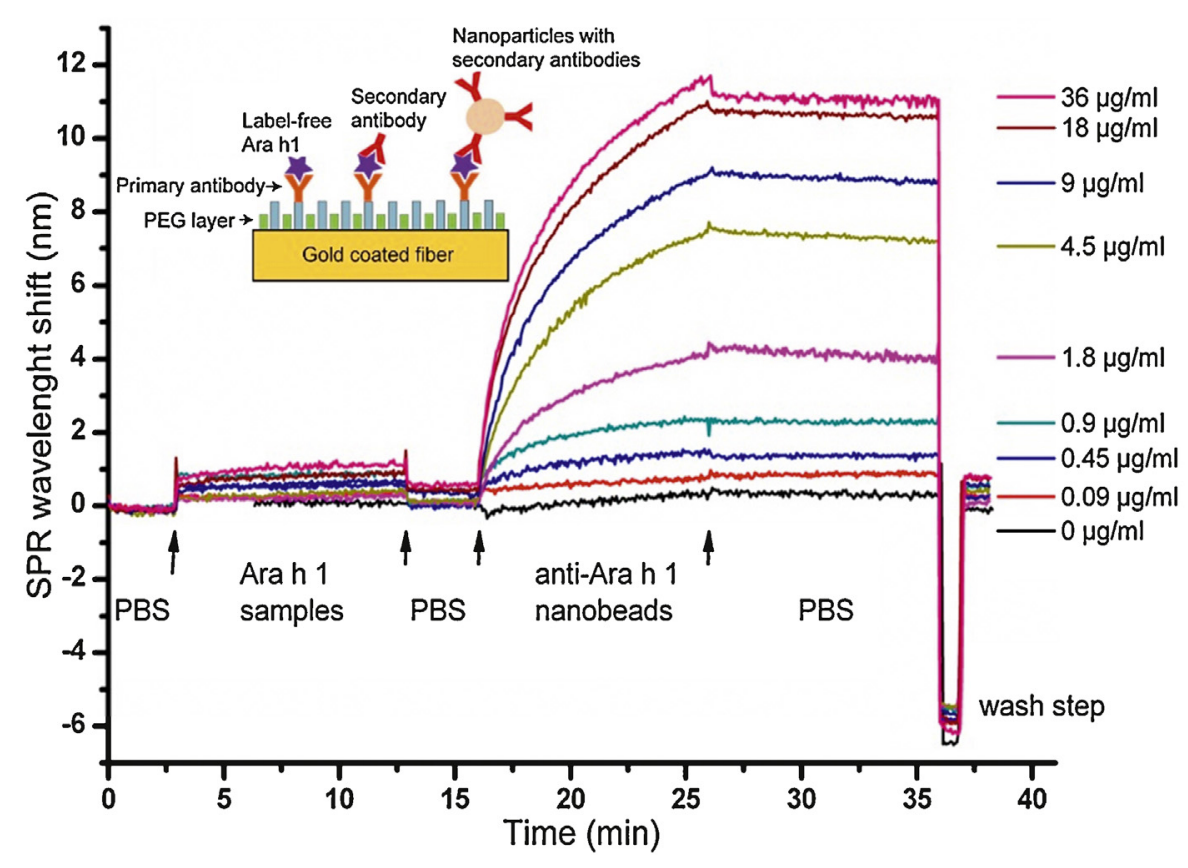

FIG. 4

Typical FO-SPR sensorgrams showing first the direct binding of different concentrations of Ara h1 peanut allergen to the sensor surface, followed by considerable signal amplification when using the magnetic NPs functionalized with polyclonal antibodies against Ara h1. The inset: schematic drawing of the corresponding "sandwich-like" bioassay implemented on the FO-SPR sensor surface. Reprinted with permission from [59]. Copyright (2011) Elsevier B.V.

prostate-specific antigen (PSA). Therefore, the authors concluded that such FO-LSPR probes can be successfully used in real-time label-free immunosensing potentially allowing for fast detection, high resolution and/or high sensitivity.

In a similar manner, the Ag mirror-coated tip of a FO (fabricated based on the Jorgenson model [37]) was modified by immobilizing $\mathrm{Au}$ NPs through interaction with the thiol groups generated during the surface silanization with (3-mercaptopropyl)trimethoxysilane (MPTMS) [63]. The SPR sensing capability was demonstrated in high-pressure environments with a sensor resolution of $1.2 \times 10^{-3} \mathrm{RIU}$, which decreased considerably with increasing the working pressure, although the sensor generated a linear response to RI changes. The same device could detect streptavidin and IgG, with a LOD in the pM range.

More recently, a positively charged polyelectrolyte (PAH) was absorbed on the negatively charged FO silica core, introducing thus amine groups for electrostatically linking metallic NPs to the FO tip [64]. This method allowed dual immobilization of both $\mathrm{Au}$ and Ag spherical NPs on the FO surface. By employing NPs with different SPR signatures and selectively functionalizing the two NP types with different antibodies, the authors demonstrated the multiplexing capability of the sensor. In this way, distinct LSPR

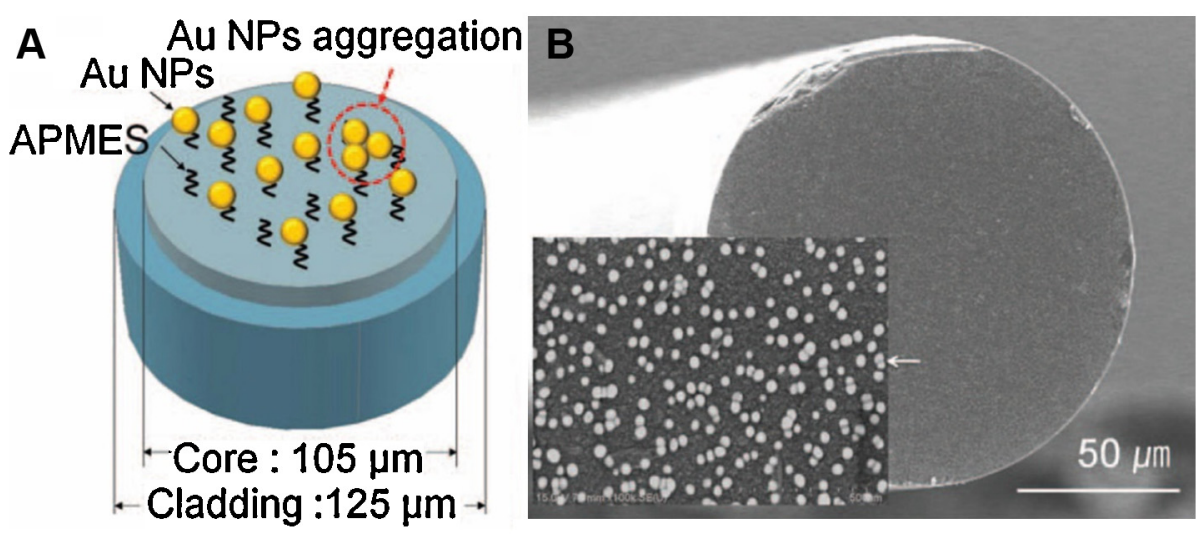

\section{FIG. 5}

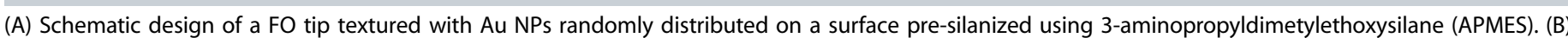
Corresponding SEM micrographs of the textured FO tip at two different magnifications. Reprinted with permission from [25]. Copyright (2011) SPIE. 
dips corresponding to two specific targets were separately monitored.

An interesting research showed that not only the morphology and size of the NPs immobilized on the FO-SPR surface may affect the sensor's sensitivity, but also the exact composition of the plasmonic nanomaterials used [65]. While immobilizing Au-Ag alloy NPs of different sizes and with two different $\mathrm{Au} / \mathrm{Ag}$ ratios, the authors observed that the Ag-rich alloy (75\% Ag and 25\% Au) NPs of $34 \mathrm{~nm}$ resulted in the highest sensitivity of $611 \mathrm{~nm} / \mathrm{RIU}$.

Concluding, the FO-LSPR sensors prepared by the methods presented in this section may be of great interest due to several unique characteristics [66], one being the multiplexing capability. The research field is still very active and many other types of nanostructures are expected to be tested for LSPR sensing in the future $[67,68]$. Nevertheless, a reproducible immobilization of NPs with uniform size distribution and regular arrangement is still challenging using any of the presented approaches, and these requirements can only be achieved using more sophisticated protocols as presented in Section 3.3.

\section{FO-SPR probes with lithographically patterned nanostructures}

The unconventional three-dimensional (3D) FO platform continuously challenges the well-established nanostructuring techniques, generally engineered and optimized to operate with planar substrates, such as the low-cost colloidal lithographic protocols [69] or the more complex top-down nanofabrication methods [70]. The nanopatterning of FO-SPR surfaces using such lithographic techniques is among the latest development in nanotechnology, particularly promising due to the versatility in the nanostructures design and functionality, paving the way toward high-performance LSPR sensing. Nanoimprinting, "nanoskiving", electron beam lithography (EBL) and focus ion beam (FIB) milling are only a few examples of advanced surface structuring methods.

The nanoimprinting is a mechanical low-cost patterning technique, which directly generates surface $3 \mathrm{D}$ profiles by physically displacing material from the substrate with the help of a compressing mold [71]. This technique was first applied to a FO tip by Kim et al. in 2001, to prepare probes for near-field scanning optical microscopy (NSOM) [72]. The authors spin-coated a polymer film onto a flat silicon ( $\mathrm{Si}$ ) wafer to create the mold by photolithography, which was then transferred to the FO tip. Following a similar approach, arrays of metallic nanostructures were fabricated and transferred to the FO tip and its circular sides using a process called "nanoskiving" [73]. Here, a block with Au-coated epoxy nanostructures was prepared for sectioning it into slabs by using a diamond-based high resolution microtome. These slabs were then transferred manually to the FO tips. In the end, the nanostructures on the FO surface were revealed by selectively etching the epoxy matrix (Fig. 6A). The technique was found extremely versatile in tuning the nanostructures geometry with demonstrated capacity for producing $\mathrm{Au}$ nanodots, rings, high aspect-ratio concentric cylinders or gratings of parallel nanowires onto the flat FO tip (Fig. 6B and C). Despite the spectacular results obtained, such probes were not tested as optical sensors, until the research group of Yang demonstrated recently the potential of the mold transferring method to design highly-performant FO-SPR sensors [74,75]. They were able to prepare periodic arrays of either 2D nanoholes or one-dimensional (1D) nanoslits. By accurately controlling the
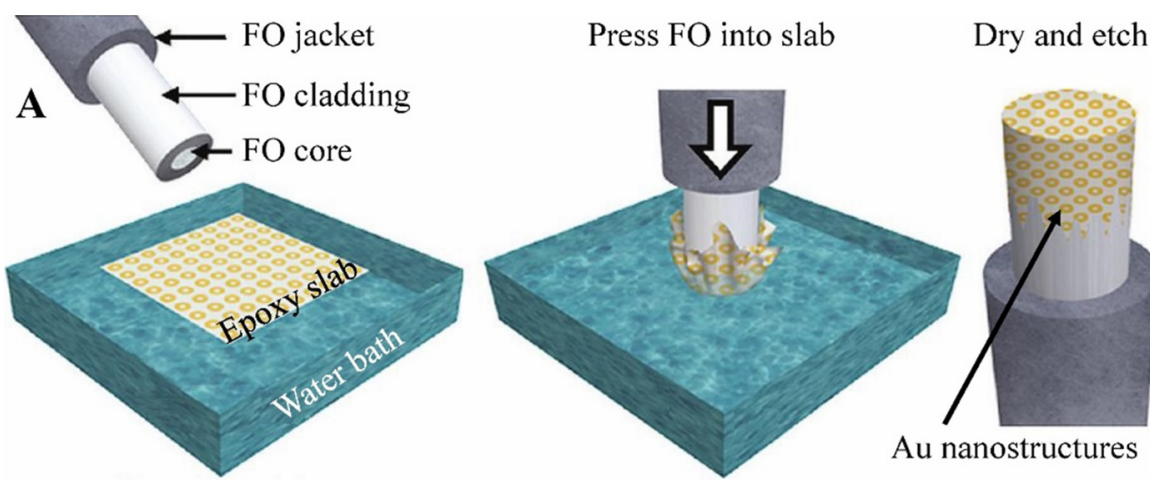

Au nanostructures
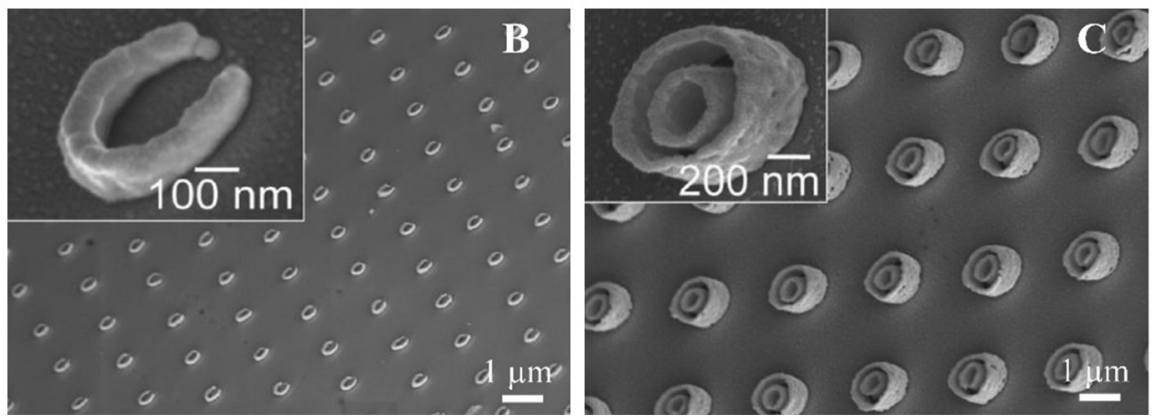

FIG. 6

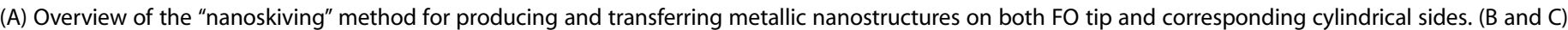
SEM micrographs showing two examples of prepared nanostructures: Au split rings (B) and an array of concentric Au rings (C). The insets are closed-up magnifications. Adapted with permission from [73]. Copyright (2011) American Chemical Society. 
preparation conditions, diverse surface topographies of these metallic structures were realized. The researchers designed further a special plasmonic FO with RI sensing capability (401 nm/RIU), coupled with a remarkably narrow resonance dip $(6.6 \mathrm{~nm})$. This resulted in a FOM of 60.7, which was four times higher than other reported values for planar plasmonic substrates patterned with $\mathrm{Au}$ nanohole arrays [76]. In addition, the authors also demonstrated the potential of this FO-SPR device for real-time immunosensing.

The nanoimprinting can be implemented also in a more straightforward manner by dispensing first a suitable polymer directly on the FO tip (i.e. by dip coating) and then using a hard mold to imprint the desired patterns into the polymer film directly on the FO surface, eliminating thus the need for a transferring step. This approach has the advantage that a pre-designed hard mold can be repeatedly used to imprint patterns on the FO substrate. The method was successfully implemented to produce Au grating diffraction elements of $20 \mathrm{~nm}$ thickness with a line periodicity of $630 \mathrm{~nm}$, by using a pre-designed $10 \times 10 \mu \mathrm{m}^{2}$ hard mold $[77,78]$. Further reductions in the size of the imprinted features were realized when employing the self-assembled nanoporous anodic aluminum oxide (AAO) as mold to design dens arrays of Ag-coated nanopillars with diameters of $70 \mathrm{~nm}$ and sub-15 nm interspacing [79]. Despite all these advancements of the nanoimprint methods in the last years, the methodology has not been yet extensively used to produce FO-SPR/LSPR biosensors. The existing reports in the literature on modifying the FO tip in this way are mostly focused on applications either in photonic integrated circuits [78] or for SERS measurements [79].

The ultimate control over the resolution, shapes, ordering and reproducibility of the obtained nanostructures is accomplished by advanced lithographic methods, such as EBL or FIB. Initially, Chen et al. made a simulation in which the authors proposed a "chessboard-like" Au pattern with nanogaps in the 6-15 nm range, lithographically drawn on a FO tip to act as a high-performance substrate for LSPR excitation [80]. Their results predicted that the local EM field of the pattern will be strongly dependent on several structural parameters of the micro-squares (i.e. length, thickness, nanogap), motivating the need of well-controlling the design of the resulted nanostructures. This interesting study challenged Lin and co-workers to use for the first time the EBL to directly pattern periodic Au nanodot arrays on the FO tips for potential applications in LSPR biochemical sensing [81]. The FO tip was first coated with a thin Au layer and then with an electron-sensitive resist. After exposing the resist surface to well-controlled electron irradiation and subsequent developing, the patterned resist was used as a mask for Au etching. In the end, the remaining resist was stripped out, exposing a nicely ordered array of $\mathrm{Au}$ nanodots on the $\mathrm{FO}$ surface (Fig. 7A). Such a FO-SPR probe was first exposed to RI measurements in various dielectric environments (i.e. air, water, acetone, etc.), demonstrating a sensitivity of $\sim 196 \mathrm{~nm} / \mathrm{RIU}$. More promising was however the employment of this probe as LSPR biosensor when implementing a bioassay based on a biotin/streptavidin model. In this case, the authors established a LOD for streptavidin of $6 \mathrm{pM}$. In a similar manner, Dhawan et al. used a FIB equipment to pattern $\mathrm{Au}$ nanohole arrays on a FO tip previously coated by 100-250 nm Au layer (Fig. 7B) [82-85]. The FO-SPR probes decorated with these interesting $\mathrm{Au}$ patterns were also tested by performing RI measurements in different solutions of alcohols, demonstrating a sensitivity of $533 \mathrm{~nm} / \mathrm{RIU}$. Furthermore, the authors clearly concluded that the sensitivity brought by these Au nanohole arrays was considerably improved compared with other FO-LSPR sensor types (i.e. based on Au NPs).

Concluding so far, the curved FO substrate can be a versatile platform for micro- and nanotechnology. However, a lot of dedicated work remains to be done, in order to develop reproducible, less complex and cheaper fabrication procedures comparable with the already established wafer-scale methods in semiconductor industry and mass-production. Furthermore, some of the achieved performance quantifiers (i.e. sensitivity) might be still modest, since the FO-LSPR systems are in a much earlier stage of development. Nevertheless, they are considered the next generation of FObased plasmonic sensors, offering tremendous possibilities for miniaturization, multiplexing and/or small molecules detection in low concentrations.

\section{Nanoparticles coupled into microstructured FO-SPR probes}

The microstructured FOs (MFOs) are the latest achievements in the FO-SPR sensing field and generally in photonics research. These types of FOs have a set of hollow channels running throughout the FO core, making the light guiding mechanism different compared

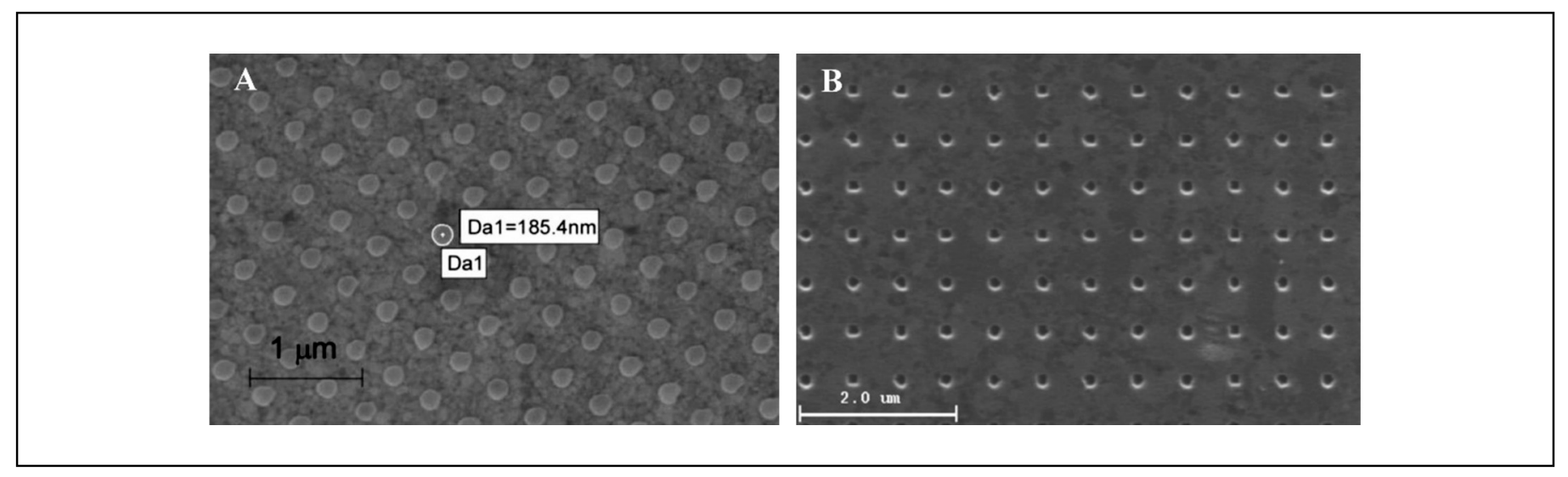

\section{FIG. 7}

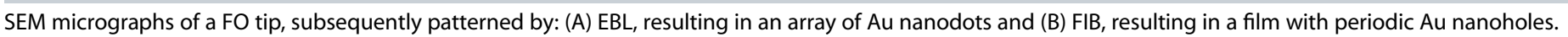
Reprinted with permissions from [81] (A) and [83] (B). Copyrights (2010) MDPI and (2008) IEEE, respectively. 
to the conventional FOs. In addition to the modified TIR mechanism, the photonic band gap created by the regular arrangement of the holes has to be carefully taken into account when considering the light guiding through a MFO [86]. In this way, very interesting applications could be revealed, especially in the field of chemical sensing and biosensing. Among the advantages of this platform are the ultra-low sample volume required and the possibility for integrating ultra-small microfluidic systems through the MFO channels, capable of transporting few nanoliters of liquid per each MFO centimeter (a preferable advantage in biosensing) [87]. The first demonstration for the detection of biomolecules using such a MFOSPR was made by Rindorf et al., when they achieved a sensitivity of $1500 \mathrm{~nm} / \mathrm{RIU}$ (at an RI value of 1.33) [88]. More recently, a special type of MFO (called "silica exposed-core MFO") was used with a small core (10 $\mu \mathrm{m}$ diameter) physically exposed along the whole FO length [89]. This feature allowed deposition of a thin plasmonic Ag film directly onto the FO core using a conventional thermal evaporation technique. Although the sensitivity of the obtained MFO-SPR device ( $2500 \mathrm{~nm} / \mathrm{RIU})$ was in the same range as for a conventional SPR sensor prepared by the authors with a large core FO (140 $\mu \mathrm{m}$ diameter), the MFO-SPR dip was three times narrower (FWHM $\sim 50 \mathrm{~nm}$ ), possibly demonstrating a better LOD, especially for detection in smaller sample volumes.

However, due to the obvious difficulties of coating uniform and consistent plasmonic films on the inner walls of MFOs channels, their coupling with metallic nanomaterials (i.e. Au NPs) is highly desirable. In this new research field, the first experiment of inserting NPs into a solid core MFO was reported by Amezcua-Correa

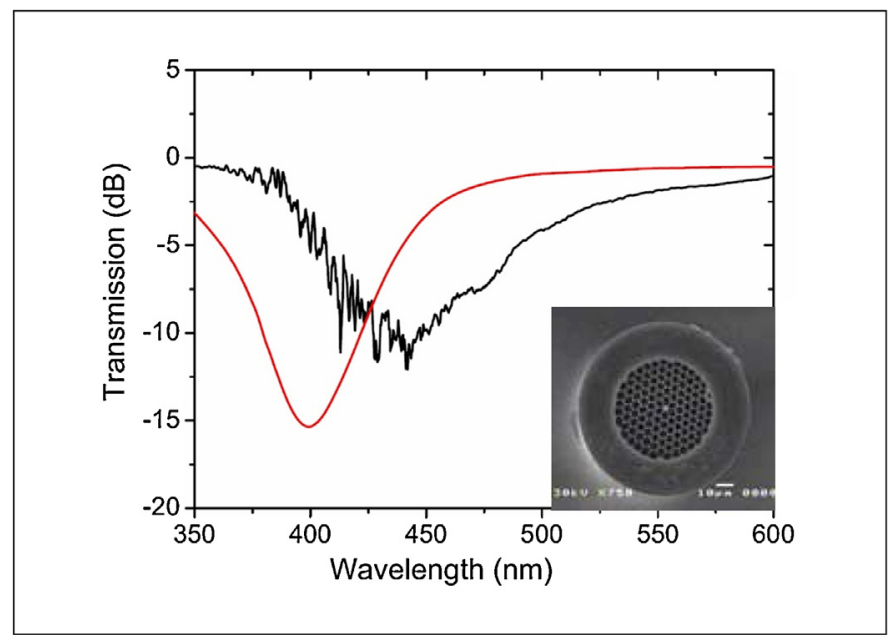

FIG. 8

SPR dip obtained when measuring a suspension containing Ag NPs (red) and the one corresponding to the Ag NPs immobilization on the MFO internal walls (black). The inset represents a SEM image of the MFO used. Reprinted with permission from [91]. Copyright (2008) IET.

et al. [90]. The authors used a special precursor containing $\mathrm{Ag}^{+}$ions and they pumped the solution through the MFO channels. The precursor was then heat-decomposed on the internal walls, resulting in Ag NPs filling the channels. Such MFO probe (see the inset of Fig. 8) was at first used only for SERS measurements, until demonstrated by de Matos et al. that it can be used for SPR sensing [91]. The researchers immobilized Au and Ag NPs inside a hollow core
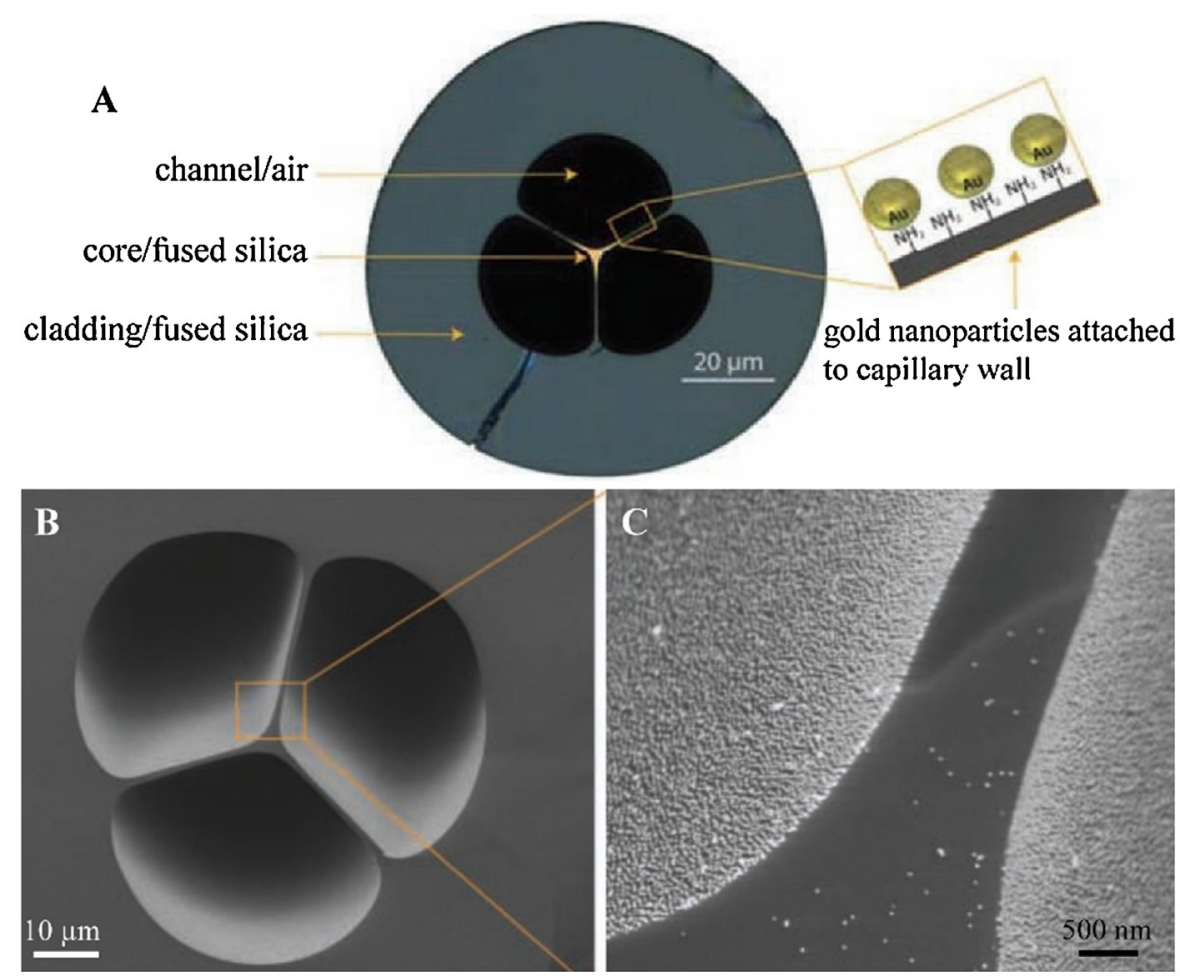

FIG. 9

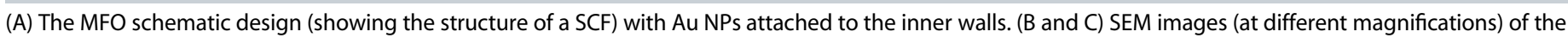
MFO inner walls coated with Au NPs (spheres with diameters of $30 \mathrm{~nm}$ ). Reprinted with permission from [92]. Copyright (2010) John Wiley \& Sons, Inc. 
TABLE 1

Performance comparison data between different FO-SPR sensing probes.

\begin{tabular}{|c|c|c|c|}
\hline FO-SPR probe fabrication procedures & $\begin{array}{l}\text { Refractive index } \\
\text { measurements }\end{array}$ & $\begin{array}{l}\text { Sensitivity } \\
\text { (nm/RIU) }\end{array}$ & $\begin{array}{l}\text { Figure of } \\
\text { merit }\left(\operatorname{RIU}^{-1}\right)\end{array}$ \\
\hline Au-coated multi-mode FO with Ag mirror on the FO tip [39] & Ethanol dilutions & 1557 & ca. 19.4 \\
\hline Tilted Bragg grating imprinted on the core of a Au-coated FO [47] & $\begin{array}{l}\text { Solutions of different } \\
\text { refractive index values }\end{array}$ & 351 & ca. 8775 \\
\hline FO-LSPR sensor with the FO tip coated by both, Au and Ag NPs [64] & Glycerol dilutions & 390 & ca. 6.5 \\
\hline Au nanoholes patterned by template transfer method on the FO tip [74] & Sodium chloride dilutions & 401 & ca. 60.7 \\
\hline Au and Ag NPs coupled into the MFO channels [89] & Glycerol dilutions & 2500 & ca. 50 \\
\hline
\end{tabular}

MFO and they observed that the SPR dip shifted when solutions of different RI values were inserted into the MFO (Fig. 8), although no surface binding results were reported.

An innovative "nanoparticle layer deposition (NLD)" technique has been also described for depositing well-defined plasmonic layers of specifically selected NPs inside the channels of a special MFO, known as "suspended core fiber (SCF)" (Fig. 9A) [92]. The method was a combination between microfluidic and self-assembled monolayer (SAM) techniques [79], and the authors demonstrated its effectiveness for obtaining a longitudinal homogeneous NPs density of several meters along the MFO (Fig. 9). This device was used as LSPR sensor, exhibiting a sensitivity of $78 \mathrm{~nm} / \mathrm{RIU}$ when carrying out RI measurements.

In conclusion, making a reliable biosensor by coupling NPs into MFOs, capable of detecting biomolecular binding events (i.e. antibody-antigen or DNA-antigen) looks like the next challenge. This exciting research field is currently fueled by many theoretical models and simulations [93-95], pointing out to the possibility of driving the SPR and LSPR sensing schemes toward new levels of miniaturization, performance, portability and applications.

\section{Conclusions}

As reflected in this short review, impressive scientific efforts were carried out lately in order to modify the FO surfaces, aiming at improved FO-SPR devices with new functionalities. Most of these efforts involve the use of nanomaterials, mainly due to the fact that their small sizes may enhance the overall performance or may enable unique effects such as LSPR, while maintaining the initial device dimensions. Table 1 gives a brief performance comparison among various FO-SPR sensors investigated by the researchers in the last years. As can be noticed, the devices FOM and sensitivity are varying considerably depending on the employed procedures for the FO-SPR probes fabrication. Therefore, the choice of the right device to use in a specific sensing scenario may be rather based on the application itself than on the actual performance of the FO-SPR platform.

Concluding, despite all these efforts, the FO-SPR technology is far from a mature sensing platform and examples of its applicability in complex chemical or biochemical sensing schemes are still limited. However, the directly related benefits of the FO-based systems, such as low cost, reduced dimensions, optical detection, material robustness, bio-compatibility and high degree of portability, ensure that the FO tip modification will remain at the forefront of the research in this area. Nevertheless, reaching the full potential of this technology still requires a much better understanding of its fundamentals, which can be achieved through an even greater cooperation between physicists, chemists, biologists and material scientists.

\section{Acknowledgments}

The authors acknowledge support and funding from the European Commission's Seventh Framework Programme (FP7/2007-2013) under the grant agreement BIOMAX (project no. 264737), from the KU Leuven Industrial Research Fund (IOF/KP/12/002 NanoDiag) and the Flemish Institute for the Promotion of Innovation through Science and Development (IWT/100936 Sensors for Food VIS-trajectory, IWT/110770 LA-trajectory and IWT/120584 Innovation mandate of F.D.).

\section{References}

[1] Wood XLII RW. On a remarkable case of uneven distribution of light in a diffraction grating spectrum. Philos Mag Ser 1902;6(4):396-402.

[2] Fano U. The theory of anomalous diffraction gratings and of quasi-stationary waves on metallic surfaces (Sommerfeld's waves). J Opt Soc Am 1941;31:213-22

[3] Rayleigh L. On the dynamical theory of gratings. Proc R Soc London Ser A 1907;79:399-416.

[4] Otto A. Excitation of nonradiative surface plasma waves in silver by the method of frustrated total reflection. Z Physik 1968;216:398-410.

[5] Kretschmann E. Die Bestimmung optischer Konstanten von Metallen durch Anregung von Oberflächenplasmaschwingungen. Z Physik 1971;241:313-24.

[6] Chen Y, Ming H. Review of surface plasmon resonance and localized surface plasmon resonance sensor. Photonic Sens 2012;2:37-49.

[7] Guo X. Surface plasmon resonance based biosensor technique: a review. J Biophotonics 2012;5:483-501.

[8] Piliarik M, Vaisocherová H, Homola J. Surface plasmon resonance biosensing. In: Rasooly A, Herold K, editors. Biosensors and biodetection. Humana Press; 2009 . p. 978-1-60327-566-865-88, http://link.springer.com/protocol/10 1007\%2F978-1-60327-567-5_5.

[9] Yuan Y, Benshun Y, Jinsheng X, ZhaoHui L. Surface plasmon resonance biosensors and its application. In: The 1st international conference on bioinformatics and biomedical engineering (ICBBE 2007). 2007. p. 1043-6.

[10] Zhang J, Zhang L, Xu W. Surface plasmon polaritons: physics and applications. J Phys D: Appl Phys 2012;45:113001.

[11] Sharma AK, Jha R, Gupta BD. Fiber-optic sensors based on surface plasmon resonance: a comprehensive review. IEEE Sens J 2007;7:1118-29.

[12] Estevez MC, Otte MA, Sepulveda B, Lechuga LM. Trends and challenges of refractometric nanoplasmonic biosensors: a review. Anal Chim Acta 2014;806:55-73.

[13] Homola J, Yee SS, Gauglitz G. Surface plasmon resonance sensors: review. Sens Actuators B: Chem 1999;54:3-15

[14] Ince R, Narayanaswamy R. Analysis of the performance of interferometry, surface plasmon resonance and luminescence as biosensors and chemosensors. Anal Chim Acta 2006;569:1-20.

[15] Jason-Moller L, Murphy M, Bruno J. Overview of BiaCore systems and their applications. In: Current protocols in protein science. John Wiley \& Sons, Inc. 20069780471140863 p. 45:19.13:19.13. 41-19.13.14http://onlinelibrary.wiley. com/doi/10.1002/0471140864.ps1913s45/ abstract;jsessionid=7B821C09AFECF3E4FA51C268840FDBD9.f01t01.

[16] Fontana E, Dulman HD, Doggett DE, Pantell RH. Surface plasmon resonance on a single mode optical fiber. IEEE Trans Instrum Meas 1998;47:168-73.

[17] Kostovski G, Stoddart PR, Mitchell A. The optical fiber tip: an inherently lightcoupled microscopic platform for micro- and nanotechnologies. Adv Mater 2014;26:3798-820. 
[18] Homola J. Present and future of surface plasmon resonance biosensors. Anal Bioanal Chem 2003;377:528-39.

[19] Boisselier E, Astruc D. Gold nanoparticles in nanomedicine: preparations, imaging, diagnostics, therapies and toxicity. Chem Soc Rev 2009;38:1759-82.

[20] Villuendas F, Pelayo J. Optical fibre device for chemical seming based on surface plasmon excitridon. Sens Actuators A: Phys 1990;23:1142-5.

[21] Dmitriev A. Nanoplasmonic sensors. In: Potyrailo RA, editor. Integrated analytical systems. New York: Springer; 2012, ISBN: 978-1-4614-3933-2, http://link. springer.com/book/10.1007\%2F978-1-4614-3933-2.

[22] Shankaran DR, Miura N. Trends in interfacial design for surface plasmon resonance based immunoassays. J Phys D: Appl Phys 2007;40:7187.

[23] Kim WM, Kim SH, Lee KS, Lee TS, Kim IH. Titanium nitride thin film as an adhesion layer for surface plasmon resonance sensor chips. Appl Surf Sci 2012;261:749-52

[24] Wang L, Li T, Du Y, Chen C, Li B, Zhou M, et al. Au NPs-enhanced surface plasmon resonance for sensitive detection of mercury(II) ions. Biosens Bioelectron 2010;25:2622-6.

[25] Jeong H-H, Erdene N, Lee S-K, Jeong D-H, Park J-H. Fabrication of fiber-optic localized surface plasmon resonance sensor and its application to detect antibody-antigen reaction of interferon-gamma. OPTICE 2011;50:124405-408.

[26] Anker JN, Hall WP, Lyandres O, Shah NC, Zhao J, Van Duyne RP. Biosensing with plasmonic nanosensors. Nat Mater 2008;7:442-53

[27] Gupta BD, Verma RK. Surface plasmon resonance-based fiber optic sensors: principle, probe designs, and some applications. J Sens 2009;2009:12

[28] Larsson Elin M, Syrenova S, Langhammer C. Nanoplasmonic sensing for nanomaterials science. In: Nanophotonics. Science Wise Publishing \& De Gruyter 2012: 249. http://dx.doi.org/10.1515/nanoph-2012-0029. http://www.degruyter.com/view/j/nanoph.2012.1.issue-3-4/nanoph-2012-0029/nanoph-20120029.xml

[29] Markatos S, Zervas MN, Giles IP. Optical fibre surface plasmon wave devices. In Electronics letters. Institution of Engineering and Technology; 1988: 287-8.

[30] Homola J, Slavík R, Čtyroký J. Interaction between fiber modes and surface plasmon waves: spectral properties. Opt Lett 1997;22:1403-5.

[31] Themistos C, Rahman BMA, Rajarajan M, Kalli K, Grattan KTV. Characterization of surface-plasmon modes in metal-clad optical waveguides. Appl Opt 2006; 45:8523-30

[32] Pollet J, Delport F, Janssen KPF, Jans K, Maes G, Pfeiffer H, et al. Fiber optic SPR biosensing of DNA hybridization and DNA-protein interactions. Biosens Bioelectron 2009·25:864-9.

[33] Zeng J, Liang D. Application of fiber optic surface plasmon resonance sensor for measuring liquid refractive index. J Intell Mater Syst Struct 2006;17:787-91.

[34] Jorgenson RC, Yee SS. A fiber-optic chemical sensor based on surface plasmon resonance. Sens Actuators B: Chem 1993;12:213-20.

[35] Lee B, Roh S, Park J. Current status of micro- and nano-structured optical fiber sensors. Opt Fiber Technol 2009;15:209-21.

[36] Obando LA, Gentleman DJ, Holloway JR, Booksh KS. Manufacture of robus surface plasmon resonance fiber optic based dip-probes. Sens Actuators B: Chem 2004;100:439-49.

[37] Jorgenson RC, Yee SS. Control of the dynamic range and sensitivity of a surface plasmon resonance based fiber optic sensor. Sens Actuators A: Phys 1994;43: $44-8$.

[38] Arghir I, Spasic D, Delport F, Lammertyn J. Improved surface plasmon resonance biosensing using silanized optical fibers. Sensors and Actuators B 2015. http:// dx.doi.org/10.1016/j.snb.2015.04.069. in revision.

[39] Suzuki H, Sugimoto M, Matsui Y, Kondoh J. Effects of gold film thickness on spectrum profile and sensitivity of a multimode-optical-fiber SPR sensor. Sen Actuators B: Chem 2008;132:26-33.

[40] Yu Y, Blake P, Roper DK. Tapered optical fibers designed for surface plasmon resonance phase matching. Langmuir 2008;25:59-63.

[41] De Maria L, Martinelli M, Vegetti G. Fiber-optic sensor based on surface plasmon interrogation. Sens Actuators B: Chem 1993;12:221-3.

[42] Obando LA, Booksh KS. Tuning dynamic range and sensitivity of white-light, multimode, fiber-optic surface plasmon resonance sensors. Anal Chem 1999;71:5116-22.

[43] Chang Y-J, Chen Y-C, Kuo H-L, Wei P-K. Nanofiber optic sensor based on the excitation of surface plasmon wave near fiber tip. BIOMEDO 2006;11:014032035 .

[44] Masson J-F, Kim Y-C, Obando LA, Peng W, Booksh KS. Fiber-optic surface plasmon resonance sensors in the near-infrared spectral region. Appl Spectrosc 2006;60:1241-6.

[45] Shevchenko Y, Francis TJ, Blair DAD, Walsh R, DeRosa MC, Albert J. In situ biosensing with a surface plasmon resonance fiber grating aptasensor. Anal Chem 2011;83:7027-34

[46] Shevchenko YY, Albert J. Plasmon resonances in gold-coated tilted fiber Bragg gratings. Opt Lett 2007:32:211-3.

[47] Caucheteur C, Shevchenko Y, Shao L-Y, Wuilpart M, Albert J. High resolution interrogation of tilted fiber grating SPR sensors from polarization properties measurement. Opt Express 2011;19:1656-64.

[48] Raether H. Surface plasmons on smooth and rough surfaces and on gratings. Berlin, Heidelberg: Springer; 2013

[49] Špačková B, Piliarik M, Kvasnička P, Themistos C, Rajarajan M, Homola J. Novel concept of multi-channel fiber optic surface plasmon resonance sensor. Sens Actuators B: Chem 2009;139:199-203.

[50] de la Escosura-Muñiz A, Parolo C, Merkoçi A. Immunosensing using nanoparticles. Mater Today 2010;13:24-34.

[51] Jans H, Huo Q. Gold nanoparticle-enabled biological and chemical detection and analysis. Chem Soc Rev 2012;41:2849-66.
[52] He L, Musick MD, Nicewarner SR, Salinas FG, Benkovic SJ, Natan MJ, et al. Colloidal Au-enhanced surface plasmon resonance for ultrasensitive detection of DNA hybridization. J Am Chem Soc 2000;122:9071-7.

[53] Tran DT, Knez K, Janssen KP, Pollet J, Spasic D, Lammertyn J. Selection of aptamers against Ara h1 protein for FO-SPR biosensing of peanut allergens in food matrices. Biosens Bioelectron 2013;43:245-51.

[54] Pollet J, Janssen KPF, Knez K, Lammertyn J. Real-time monitoring of solid-phase PCR using fiber-optic SPR. Small 2011;7:1003-6.

[55] Knez K, Janssen KPF, Pollet J, Spasic D, Lammertyn J. Fiber-optic high-resolution genetic screening using gold-labeled gene probes. Small 2012;8:868-72.

[56] Knez K, Janssen KPF, Spasic D, Declerck P, Vanysacker L, Denis C, et al. Spherical nucleic acid enhanced FO-SPR DNA melting for detection of mutations in Legionella pneumophila. Anal Chem 2013;85:1734-42.

[57] Delport F, Pollet J, Janssen K, Verbruggen B, Knez K, Spasic D, et al. Real-time monitoring of DNA hybridization and melting processes using a fiber optic sensor. Nanotechnology 2012;23:065503.

[58] Hsieh B-Y, Chang Y-F, Ng M-Y, Liu W-C, Lin C-H, Wu H-T, et al, Localized surface plasmon coupled fluorescence fiber-optic biosensor with gold nanoparticles. Anal Chem 2007;79:3487-93

[59] Pollet J, Delport F, Janssen KPF, Tran DT, Wouters J, Verbiest T, et al. Fast and accurate peanut allergen detection with nanobead enhanced optical fiber SPR biosensor. Talanta 2011;83:1436-41

[60] Mullen KI, Carron KT. Surface-enhanced Raman spectroscopy with abrasively modified fiber optic probes. Anal Chem 1991;63:2196-9.

[61] Meriaudeau F, Wig A, Passian A, Downey T, Buncick M, Ferrell TL. Gold island fiber optic sensor for refractive index sensing. Sens Actuators B: Chem 2000;69:51-7.

[62] Mitsui K, Handa Y, Kajikawa K. Optical fiber affinity biosensor based on localized surface plasmon resonance. Appl Phys Lett 2004;85:4231-3.

[63] Lin T-J, Lou C-T. Reflection-based localized surface plasmon resonance fiberoptic probe for chemical and biochemical sensing at high-pressure conditions. J Supercrit Fluids 2007;41:317-25

[64] Sciacca B, Monro TM. Dip biosensor based on localized surface plasmon resonance at the tip of an optical fiber. Langmuir 2014;30:946-54

[65] Tu H, Sun T, Grattan KT. SPR-based optical fiber sensors using gold-silver alloy particles as the active sensing material. IEEE Sens J 2013;13:2192-9.

[66] Stewart ME, Anderton CR, Thompson LB, Maria J, Gray SK, Rogers JA, et al Nanostructured plasmonic sensors. Chem Rev 2008;108:494-521.

[67] Chen C-H, Tsao T-C, Li W-Y, Shen W-C, Cheng C-W, Tang J-L, et al. Novel Ushape gold nanoparticles-modified optical fiber for localized plasmon resonance chemical sensing. Microsyst Technol 2010;16:1207-14.

[68] Shao Y, Xu S, Zheng X, Wang Y, Xu W. Optical fiber LSPR biosensor prepared by gold nanoparticle assembly on polyelectrolyte multilayer. Sensors 2010;10: 3585-96.

[69] Henry A-I, Bingham JM, Ringe E, Marks LD, Schatz GC, Van Duyne RP. Correlated structure and optical property studies of plasmonic nanoparticles. J Phys Chem C 2011;115:9291-305.

[70] Valsecchi C, Brolo AG. Periodic metallic nanostructures as plasmonic chemical sensors. Langmuir 2013:29:5638-49.

[71] Hua F, Sun Y, Gaur A, Meitl MA, Bilhaut L, Rotkina L, et al. Polymer imprint lithography with molecular-scale resolution. Nano Lett 2004;4:2467-71.

[72] Kim BJ, Flamma JW, Ten Have ES, Garcia-Parajo MF, Van Hulst NF, Brugger J. Moulded photoplastic probes for near-field optical applications. J Microsc 2001;202:16-21.

[73] Lipomi DJ, Martinez RV, Kats MA, Kang SH, Kim P, Aizenberg J, et al. Patterning the tips of optical fibers with metallic nanostructures using nanoskiving. Nano Lett 2010;11:632-6.

[74] Jia P, Yang J. A plasmonic optical fiber patterned by template transfer as a high performance flexible nanoprobe for real-time biosensing. Nanoscale 2014:6:8836-43.

[75] Jia P, Yang J. Integration of large-area metallic nanohole arrays with multimode optical fibers for surface plasmon resonance sensing. Appl Phys Lett 2013;102:243107.

[76] Henzie J, Lee MH, Odom TW. Multiscale patterning of plasmonic metamaterials. Nat Nanotechnol 2007;2:549-54. http://dx.doi.org/10.1038/nnano.2007.252 http://www.nature.com/nnano/journal/v2/n9/full/nnano.2007.252.html.

[77] Scheerlinck S, Dubruel P, Bienstman P, Schacht E, Van Thourhout D, Baets R. Metal grating patterning on fiber facets by UV-based nano imprint and transfer lithography using optical alignment. J Lightwave Technol 2009;27:1415-20.

[78] Scheerlinck S, Taillaert D, Van Thourhout D, Baets R. Flexible metal grating based optical fiber probe for photonic integrated circuits. Appl Phys Lett 2008:92:031104.

[79] Kostovski G, Chinnasamy U, Jayawardhana S, Stoddart PR, Mitchell A. Sub $15 \mathrm{~nm}$ optical fiber nanoimprint lithography: a parallel, self-aligned and portable approach. Adv Mater 2011;23:531-5.

[80] Chen S, Han L, Schülzgen A, Li H, Li L, Moloney JV, et al. Local electric field enhancement and polarization effects in a surface-enhanced Raman scattering fiber sensor with chessboard nanostructure. Opt Express 2008:16:13016-23.

[81] Lin Y, Zou Y, Mo Y, Guo J, Lindquist RG. E-beam patterned gold nanodot arrays on optical fiber tips for localized surface plasmon resonance biochemical sensing. Sensors (Basel) 2010;10:9397-406.

[82] Dhawan A, Gerhold M, Madison A, Fowlkes J, Russell PE, Vo-Dinh T, et al. Fabrication of nanodot plasmonic waveguide structures using FIB milling and electron beam-induced deposition. Scanning 2009;31:139-46.

[83] Dhawan A, Gerhold MD, Muth JF. Plasmonic structures based on subwavelength apertures for chemical and biological sensing applications. IEEE Sens 2008;8:942-50. 
[84] Dhawan A, Muth JF. Engineering surface plasmon based fiber-optic sensors. Mater Sci Eng B 2008;149:237-41.

[85] Dhawan A, Muth JF, Leonard DN, Gerhold MD, Gleeson J, Vo-Dinh T, et al. Focused ion beam fabrication of metallic nanostructures on end faces of optical fibers for chemical sensing applications. J Vac Sci Technol B 2008;26:2168-73.

[86] Zolla F, Renversez G, Nicolet A, Kuhlmey B, Guenneau S, Felbacq D. Foundations of Photonic Crystal Fibres. In: Foundations of Photonic Crystal Fibres. 2005;9781848167285376. http://www.worldscientific.com/worldscibooks/10.1142/ p367.

[87] Fan X, White IM, Shopova SI, Zhu H, Suter JD, Sun Y. Sensitive optical biosensors for unlabeled targets: a review. Anal Chim Acta 2008;620:8-26.

[88] Rindorf L, Bang O. Highly sensitive refractometer with a photonic-crystal-fiber long-period grating. Opt Lett 2008;33:563-5.

[89] Klantsataya E, François A, Zuber A, Torok V, Kostecki R, Monro TM. Exposed core microstructured optical fiber surface plasmon resonance biosensor. Proc SPIE 2014;8938. http://dx.doi.org/10.1117/12.2039336, http://proceedings. spiedigitallibrary.org/proceeding.aspx?articleid=1833915.
[90] Amezcua-Correa A, Yang J, Finlayson CE, Peacock AC, Hayes JR, Sazio PJA, et al. Surface-enhanced Raman scattering using microstructured optical fiber substrates. Adv Funct Mater 2007;17:2024-30.

[91] de Matos CJS, Cordeiro CMB, Andrade GFS, Brolo AG, Brito-Silva AM, Temperini M.L.A.. et al. Creating and fixing a metal nanoparticle layer on the holes of microstructured fibers for plasmonic applications. In: Conference on lasers and electro-optics, 2008 and 2008 conference on quantum electronics and laser science (CLEO/QELS 2008). 2008;1-2

[92] Csaki A, Jahn F, Latka I, Henkel T, Malsch D, Schneider T, et al. Nanoparticle layer deposition for plasmonic tuning of microstructured optical fibers. Small 2010;6:2584-9

[93] Florous NJ, Saitoh K, Koshiba M. Numerical modeling of cryogenic temperature sensors based on plasmonic oscillations in metallic nanoparticles embedded into photonic crystal fibers. IEEE Photonics Technol Lett 2007;19:324-6.

[94] Hassani A, Skorobogatiy M. Design criteria for microstructured-optical-fiberbased surface-plasmon-resonance sensors. J Opt Soc Am B 2007;24:1423-9.

[95] Hautakorpi M, Mattinen M, Ludvigsen H. Surface-plasmon-resonance sensor based on three-hole microstructured optical fiber. Opt Express 2008;16:8427-32. 